10-1-1991

\title{
Internal Caustic Structure of Illuminated Liquid Droplets
}

James A. Lock

Cleveland State University, j.lock@csuohio.edu

Edward A. Hovenac

Follow this and additional works at: https://engagedscholarship.csuohio.edu/sciphysics_facpub

Part of the Physics Commons

How does access to this work benefit you? Let us know!

\section{Publisher's Statement}

This paper was published in Journal of the Optical Society of America A: Optics Image Science and Vision and is made available as an electronic reprint with the permission of OSA. The paper can be found at the following URL on the OSA website: http://www.opticsinfobase.org/josaa/ abstract.cfm?URI=josaa-8-10-1541. Systematic or multiple reproduction or distribution to multiple locations via electronic or other means is prohibited and is subject to penalties under law.

\section{Original Citation}

Lock, James A. and Edward A. Hovenac. "Internal Caustic Structure of Illuminated Liquid Droplets." Journal of the Optical Society of America A: Optics Image Science and Vision 8 (1991): 1541-1553.

\section{Repository Citation}

Lock, James A. and Hovenac, Edward A., "Internal Caustic Structure of Illuminated Liquid Droplets" (1991). Physics Faculty Publications. 101.

https://engagedscholarship.csuohio.edu/sciphysics_facpub/101

This Article is brought to you for free and open access by the Physics Department at EngagedScholarship@CSU. It has been accepted for inclusion in Physics Faculty Publications by an authorized administrator of EngagedScholarship@CSU. For more information, please contact library.es@csuohio.edu. 


\title{
Internal caustic structure of illuminated liquid droplets
}

\author{
James A. Lock \\ Department of Physics, Cleveland State University, Cleveland, Ohio 44115
}

Edward A. Hovenac

Sverdrup Technology, Inc., NASA Lewis Research Center, Cleveland, Ohio 44135

Received December 10, 1990; revised manuscript received May 9, 1991; accepted May 9, 1991

\begin{abstract}
The internal electric field of an illuminated liquid droplet is studied in detail with the use of both wave theory and ray theory. The internal field attains its maximum values on the caustics within the droplet. Ray theory is used to determine the equations of these caustics and the density of rays on them. The Debye-series expansion of the interior-field Mie amplitudes is used to calculate the wave-theory version of these caustics. The physical interpretation of the sources of stimulated Raman scattering and fluorescence emission within a liquid droplet is then given.
\end{abstract}

\section{INTRODUCTION}

When a spherical liquid droplet is illuminated by a light source, the light is partially focused inside the droplet, forming patterns of caustic lines. Our purpose in this paper is to analyze these patterns by using a reformulation of Mie theory. One limitation of Mie theory is that it often gives little physical insight as to the origins of various scattering effects. As an alternative, ray theory often provides physical insight into the scattering process. However, ray calculations are imcomplete and do not take into account wavelike phenomena such as diffraction and interference. The gap between Mie theory and ray theory can be bridged with the use of the Debye-series expansion. The Debye series rearranges the Mie equations into an infinite series of internal reflection and transmission terms. Since the Debye series is mathematically equivalent to the Mie equations, it is exact and accounts for all the mechanisms that produce scattering. Its usefulness comes from the calculation of the individual terms of the series because they are analogous to reflected and transmitted light rays. These terms of the Debye series provide the physical insight into the origin of the caustic patterns inside the spherical droplet.

This paper represents the first time to our knowledge that the Debye series has been used to study in detail the interior scattering pattern of illuminated liquid droplets. In this paper we also make use of the full Mie theory, as well as ray theory, to analyze the internal caustic structure of a liquid droplet. The results of stimulated Raman scattering (SRS) and fluorescence emission experiments are investigated as well, and the physical mechanisms that produce the sources of SRS and fluorescence in the droplet are determined with the use of the above analytical methods.

\section{RAY THEORY OF THE CAUSTICS WITHIN A LIQUID DROPLET}

In this section we consider a spherical droplet of radius $a$ and refractive index $n$ whose center is at the origin of the coordinate system and which is illuminated by a family of rays propagating parallel to the positive $Z$ axis. This geometry is shown in Fig. 1. Consider a ray whose angle of incidence at the droplet is $\theta_{i}$. The fraction of this ray's intensity that is transmitted into the droplet is $T\left(\theta_{i}\right)$, and the fraction that is reflected at the surface is $R\left(\theta_{i}\right)$. For $a \gg \lambda$ the droplet surface is approximately flat; thus $T\left(\theta_{i}\right)$ and $R\left(\theta_{i}\right)$ are given by the squares of the Fresnel coefficients. ${ }^{1}$ For the ray that is transmitted into the droplet, the fraction of its intensity that is internally reflected at each subsequent interaction with the droplet surface is also $R\left(\theta_{i}\right)$.

We define the $p$-ray family to be those rays within the droplet for all $\theta_{i}$ that have made $p-1$ internal reflections. For example, when the incident rays are transmitted into the droplet they become the $p=1$ rays, after their first reflection inside the droplet they become the $p=2$ rays, etc. Figure 2 shows several families of rays (line drawings) for $p$ values ranging from 1 to 8 . The value for the index of refraction is taken as $n=1.36+0 i$ (i.e., an ethanol droplet), and the incident light is traveling from left to right in the figure. The line drawings of Fig. 2 show many different caustics. These drawings are two-dimensional sections of the spherical droplet. The full ray picture is given by a figure of revolution about the $Z$ axis for each drawing. Thus the points where the $p=1$ caustic intersects the droplet surface (on the shadow side) is a caustic ring when the figure is rotated. A photograph of the $p=1$ caustic ring on 18.4-cm-diameter thin-walled spherical glass globe filled with water is shown as Fig. 3(a). The $p=2$ caustic in Fig. 2 is a cusp of revolution whose focal point is on the $Z$ axis at $r=0.61 a$ when $n=1.36$. A photograph of the $p=2$ cusp of revolution for the thinwalled water-filled glass globe is shown as Fig. 3(b). For each higher value of $p$, the caustic is also a cusp of revolution that winds further and further around the sphere and intersects itself on the axis a number of times. These caustics will be referred to as the cusp caustics of the droplet. 


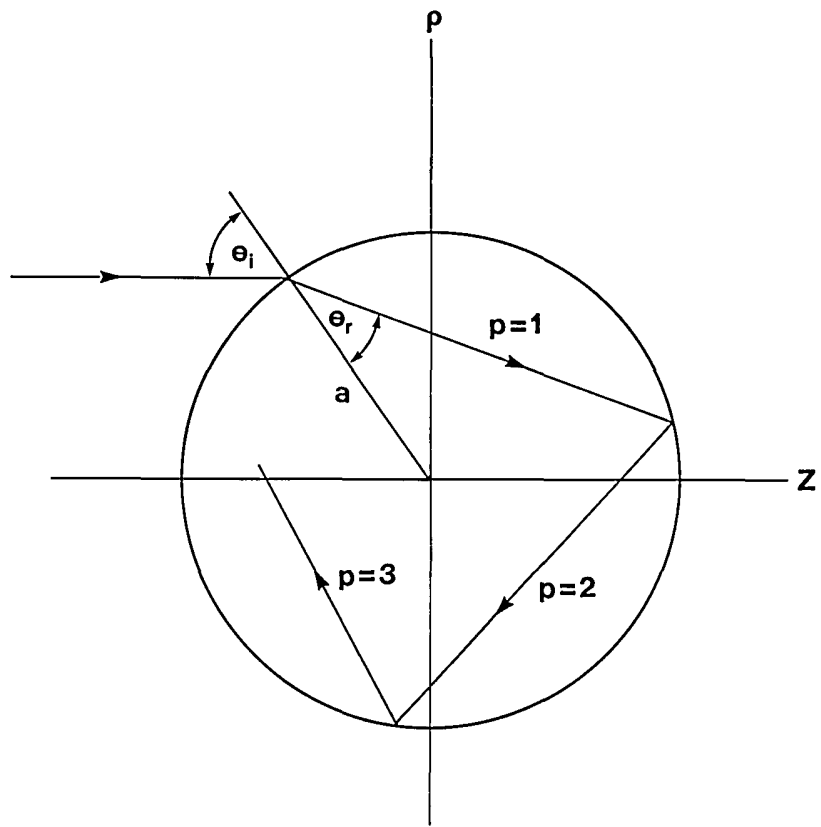

Fig. 1. Geometrical light ray with an angle of incidence $\theta_{i}$ entering and internally reflecting within a liquid droplet of radius $a$.

Employing the definition of a caustic as the envelope of the $p$-ray family, ${ }^{2}$ we find in Appendix A that the coordinates of the $p$-ray cusp caustic are

$$
\begin{aligned}
& z=\left[-\sin (\gamma) \sin \left(\theta_{r}\right)+\kappa \cos (\gamma) \cos \left(\theta_{r}\right)\right] a, \\
& \rho=\left[-\cos (\gamma) \sin \left(\theta_{r}\right)-\kappa \sin (\gamma) \cos \left(\theta_{r}\right)\right] a,
\end{aligned}
$$

where, from Fig. 1, $\rho$ is the distance perpendicular to the $Z$ axis, $\theta_{r}$ is the angle of refraction of the ray whose angle of incidence is $\theta_{i}$, and

$$
\begin{aligned}
n \sin \left(\theta_{r}\right) & =\sin \left(\theta_{i}\right), \\
\gamma & =\theta_{i}+p \pi-(2 p-1) \theta_{r}, \\
\kappa & =\left[2 p-1-n \frac{\cos \left(\theta_{r}\right)}{\cos \left(\theta_{i}\right)}\right]^{-1} .
\end{aligned}
$$

The cusp points of the caustics of Fig. 2 have the coordinates

$$
\begin{aligned}
& z_{c}=\frac{(-1)^{p}}{2 p-1-n} a, \\
& \rho_{c}=0
\end{aligned}
$$

and, near a cusp point, the cusp takes the usual Pearcey form $^{3}$

$$
\left(z-z_{c}\right) / a=W(\rho / a)^{2 / 3}
$$

where

$$
\begin{aligned}
& W=\frac{(-1)^{p}}{2} \\
& \times\left\{\frac{9\left[(2 p-1-n)^{3}-(2 p-1-n)+n\left(n^{2}-1\right)\right]}{(2 p-1-n)^{4}}\right\}^{1 / 3} .
\end{aligned}
$$

Since the caustics of Fig. 2 and Eqs. (1) correspond to $p$ rays transmitted into and reflecting within a single cross section of the sphere, the equations of the analogous raytheory caustics for a circular cylinder illuminated by normally incident rays are identical.

In addition to the cusp caustic, a second type of caustic that is not readily apparent in Fig. 2 also exists within the droplet. It is the axial caustic. This caustic corresponds to the focusing on the $Z$ axis of light rays that enter the sphere along a ring of constant $\theta_{i}$. Thus, for any $p$ family of rays, an axial caustic will be present if the $p$ rays cross the $Z$ axis. For the droplet depicted in Fig. 2, it can be seen that $p$ values of two and larger have the required internal-axis crossing that forms the axial caustic. Also note in Fig. 2 that the axial caustic never extends across the full length of the droplet's axis, since rays never cross the $Z$ axis near the center of the droplet.

The coordinates of the $p$-ray axial caustic within the sphere,

$$
\begin{aligned}
& z=-a \frac{\sin \left(\theta_{r}\right)}{\sin (\gamma)}, \\
& \rho=0,
\end{aligned}
$$

are calculated in Appendix A. A photograph of the internal $p=2$ axial caustic for the thin-walled water-filled glass globe is shown as Fig. 3(c). For $\theta_{i}=0$, the $p$-ray axial caustic begins at the cusp point of Eqs. (5). For increasing $\theta_{i}$ it proceeds along the axis inside the cusp of revolution toward the surface. For large $p$ it continues back and forth along the droplet axis with turning points at the locations where the cusp caustic intersects itself. This behavior is shown in Fig. 4 for the $p=5$ axial caustic. In the figure the incident rays are labeled with the letters A-P, and the crossing point of each ray on the axis is labeled with the same letter. The exiting rays are denoted with primes and are shown so that the direction of travel of the ray can be seen. Note that in the figure there are regions in which the axis crossings by the $p$ rays are denser: points $A-D$ and $K-N$. It is within the second of these regions that the turning point $(L)$ of the axial caustic occurs. Also note that some of the $p=5$ rays do not cross the axis. These are rays that enter the droplet at angles greater than the entering angle of ray $\mathrm{H}$ and less than the entering angle of ray I and also rays that enter at angles greater than the entering angle of ray $P$.

One important difference between the axial caustics and the cusp caustics is the degree of focusing that each represents. For the cusp caustics, incident rays that enter the droplet over a small element of the droplet's surface area contribute to the caustic over a small element of the caustic's surface area. For the axial caustics, incident rays entering the droplet over an element of the droplet's surface area (in this case it is in the shape of an annular ring) contribute to the axial caustic over an element of the axial caustic's one-dimensional length. This mapping (surface $\rightarrow$ line) for the axial caustic corresponds to a higher degree of focusing than does the mapping (surface $\rightarrow$ surface) for the cusp caustics of revolution. As a result, the axial caustics are expected to be the largest contributor to the intensity on the droplet axis. The turning points of the axial caustics represent an even higher degree of focusing, since at these points incident 
$p=1$

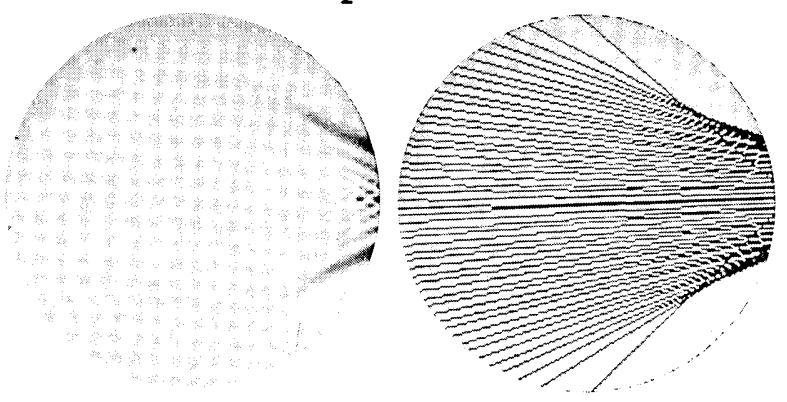

$p=3$
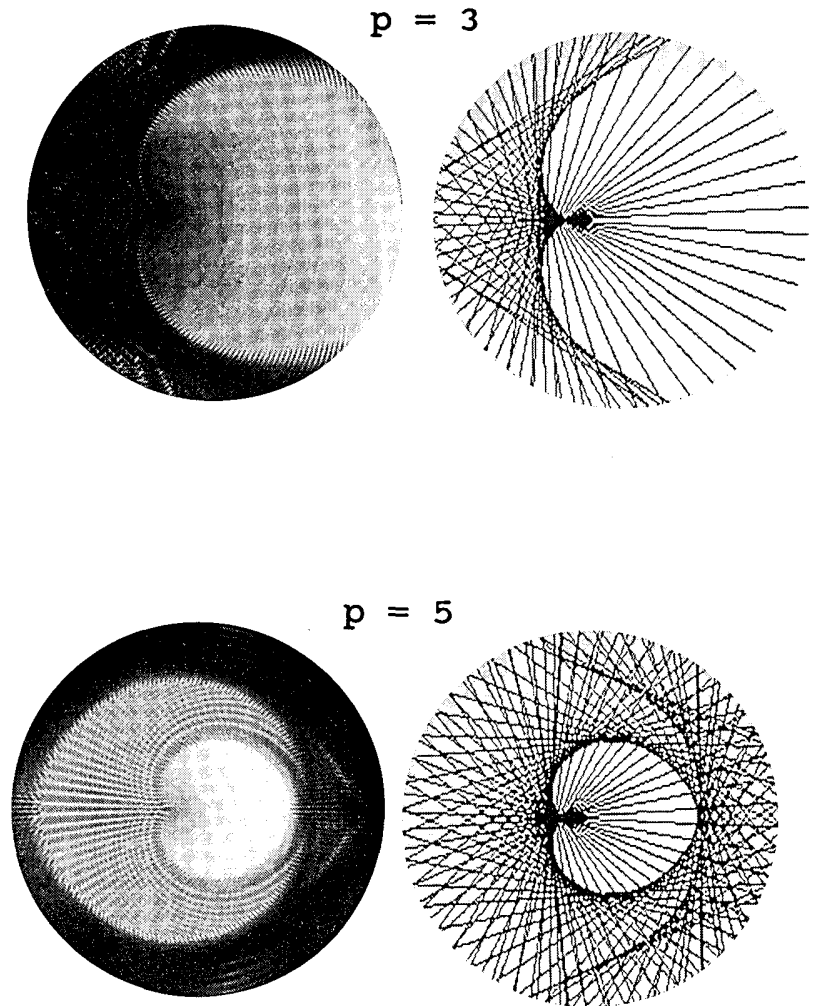

$=5$

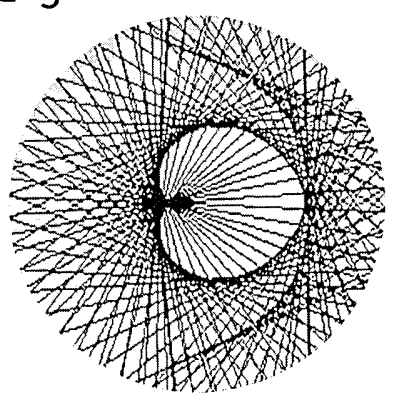

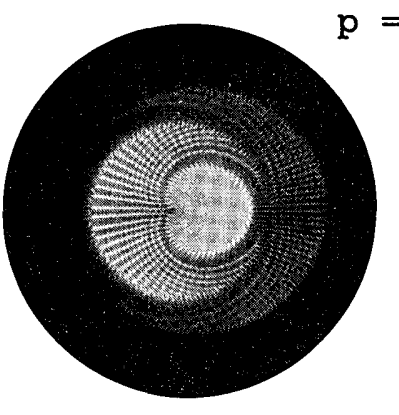

WAVE THEORY

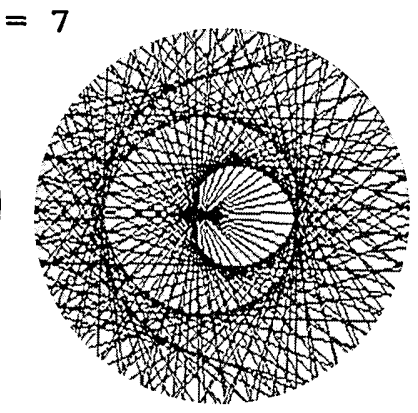

RAY THEORY

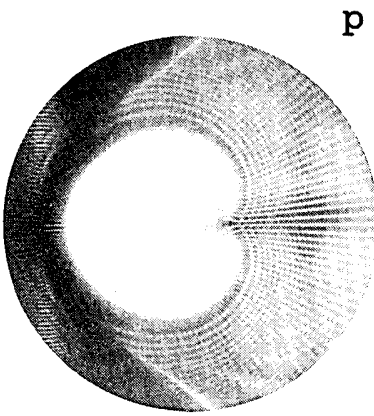

$\mathrm{p}=2$
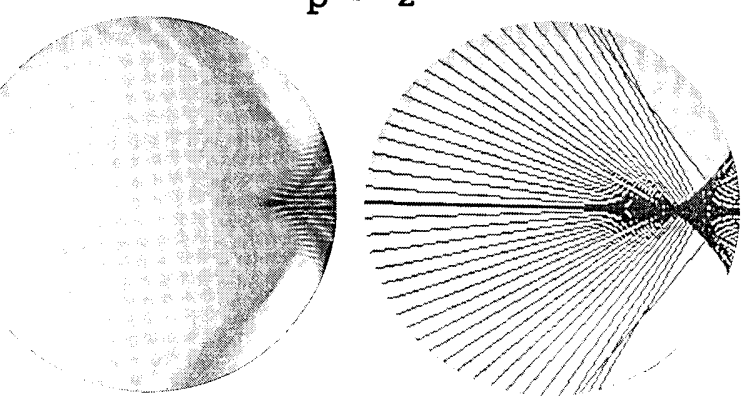

$p=4$
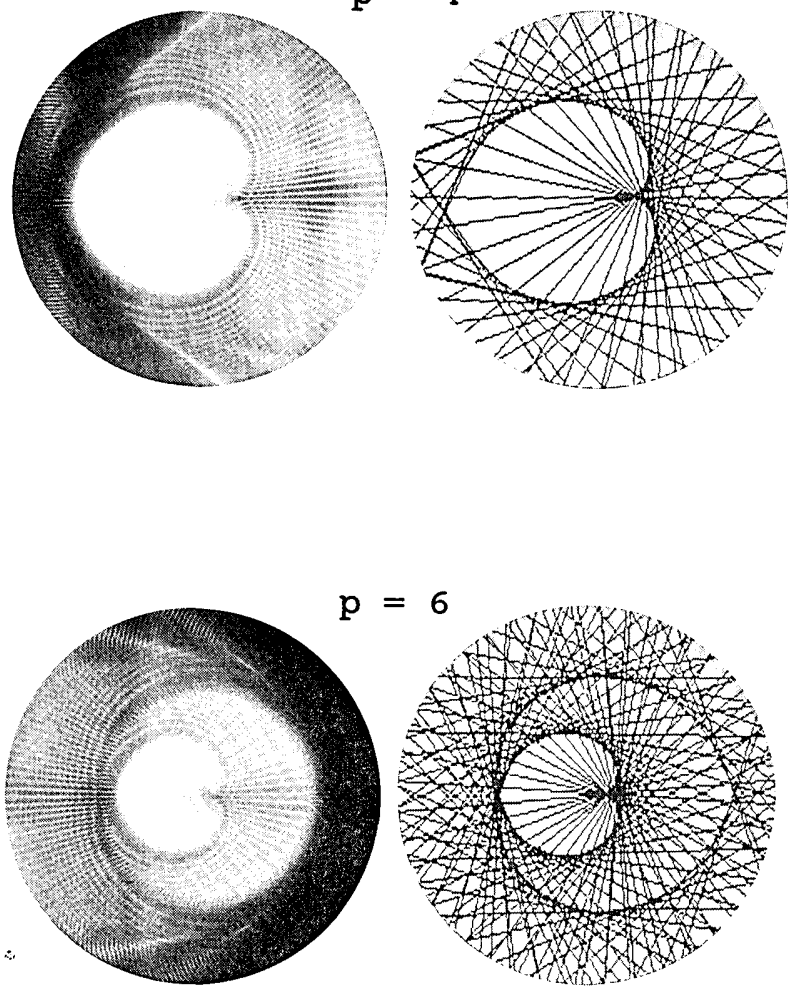

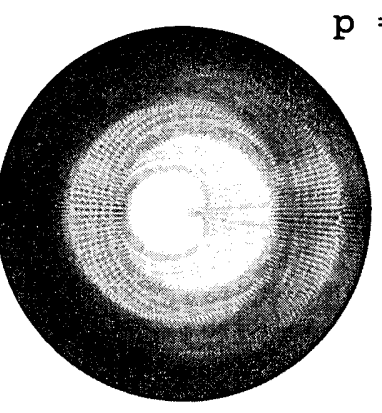

WAVE THEORY

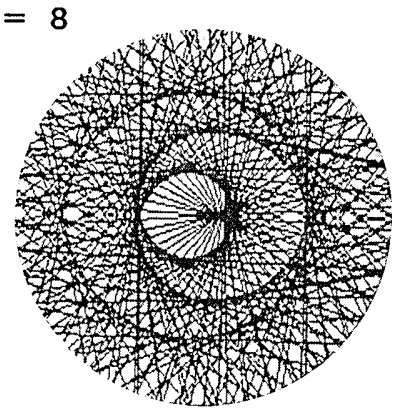

RAY THEORY

Fig. 2. Gray-level plots (left-hand side) of the $p$ term of the Debye-series interior source function for $x=100$ and $n=1.36$ for unpolarized incident light. Line drawings (right-hand side) of the geometrical $p$-ray family within the droplet for $n=1.36$. 


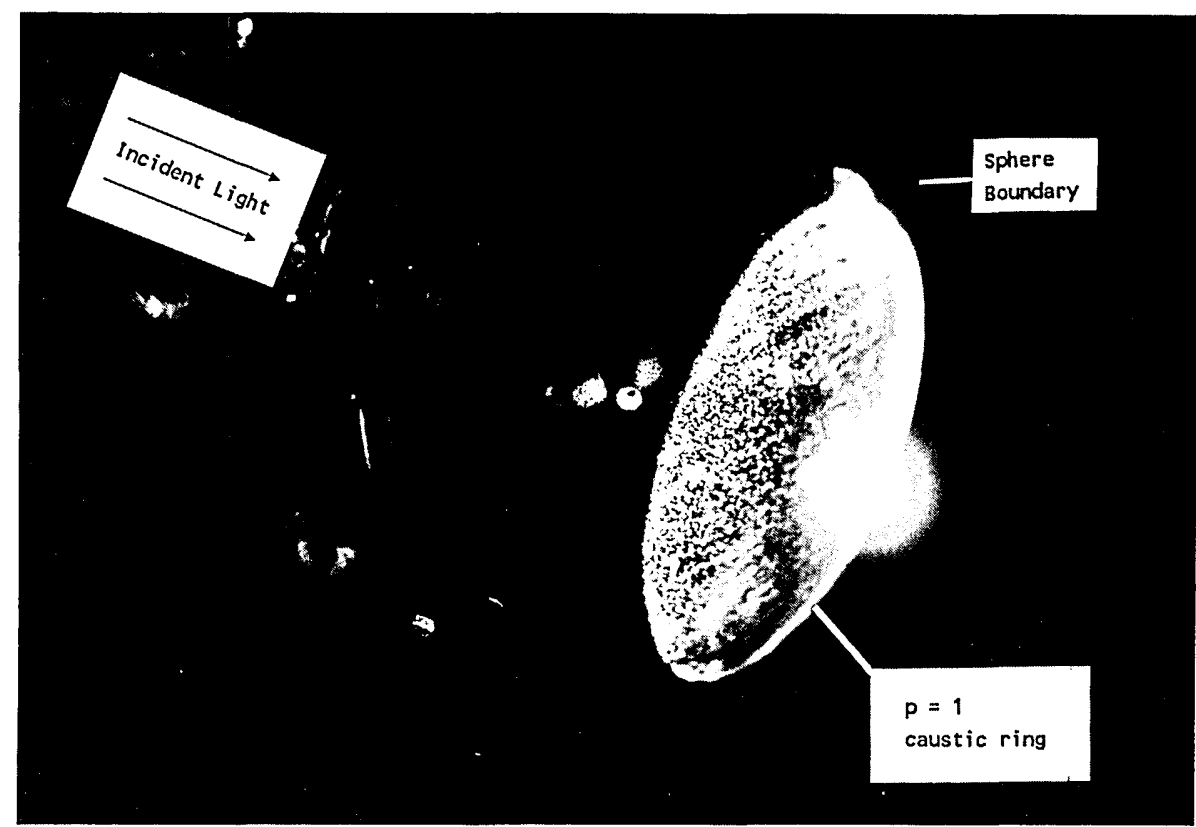

(a)

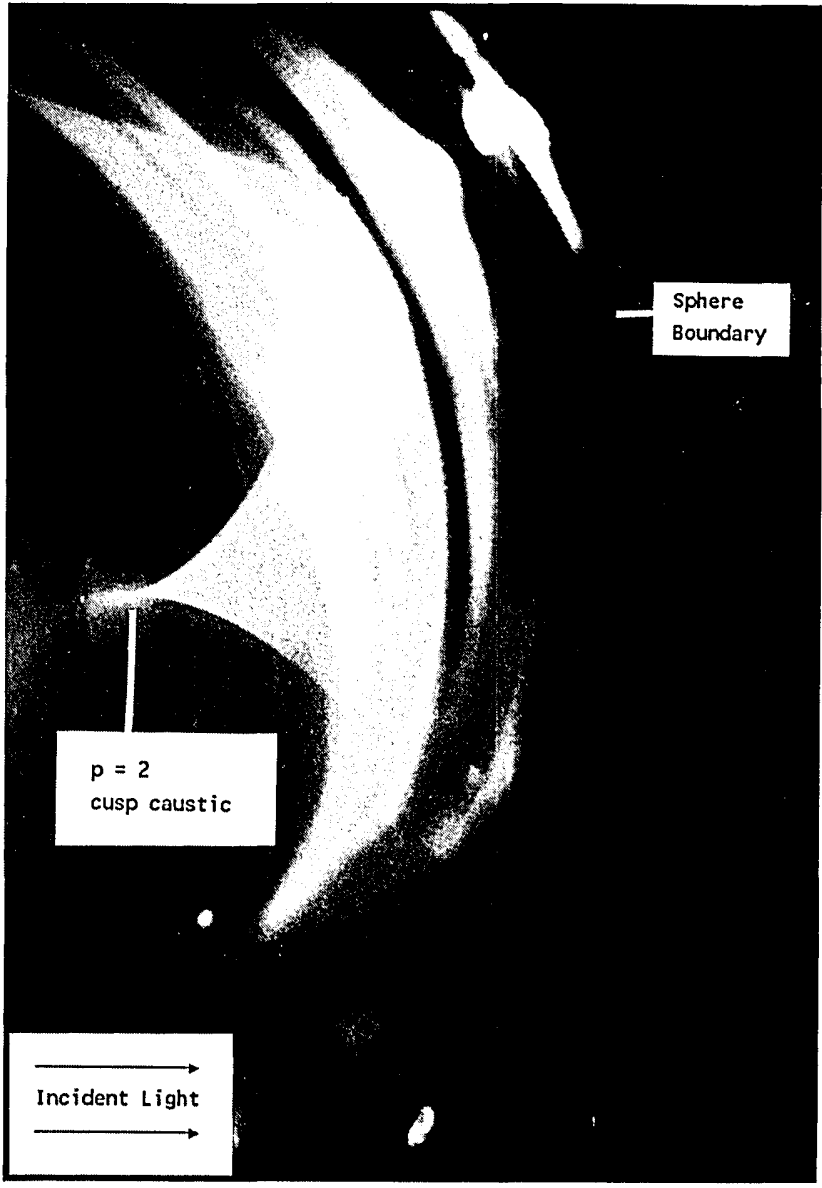

(b)

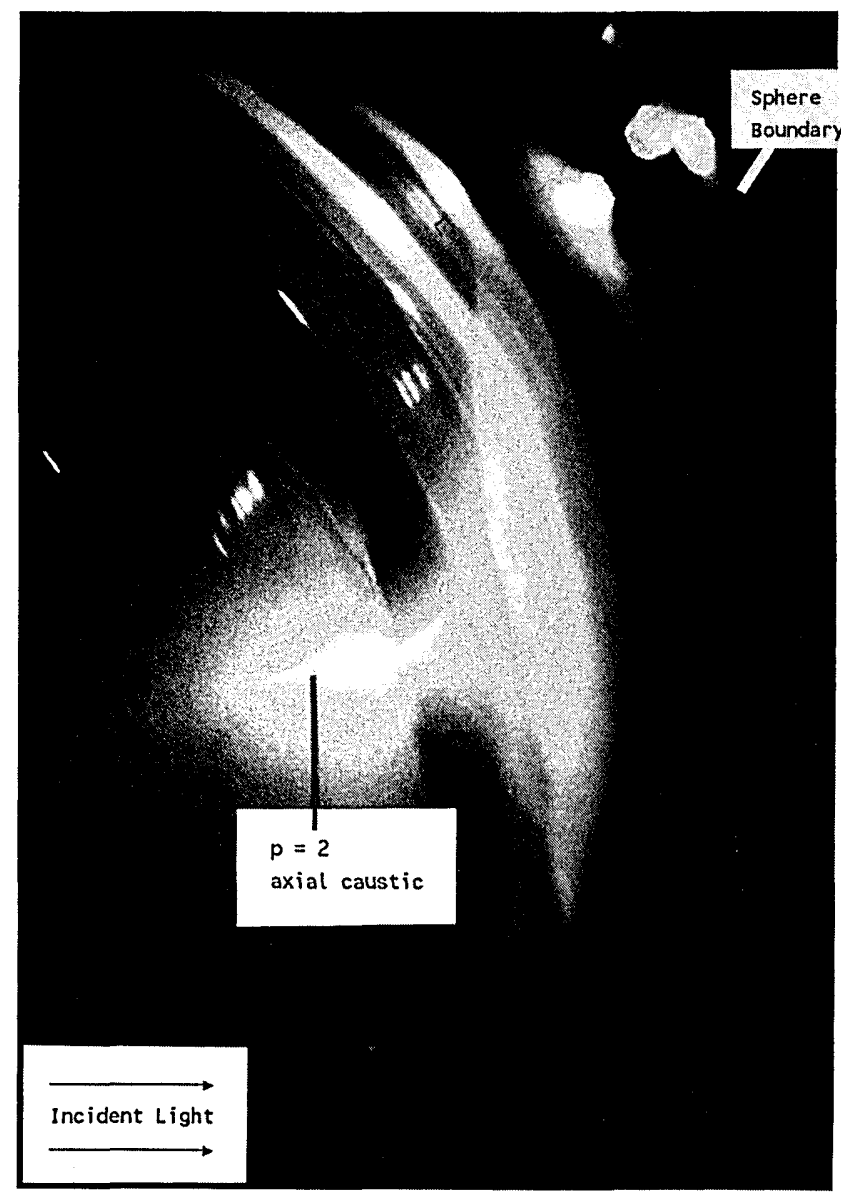

(c)

Fig. 3. 18.4-cm thin-walled water-filled spherical glass globe: (a) the $p=1$ caustic ring, (b) the $p=2$ cusp caustic of revolution, (c) the $p=2$ axial caustic.

rays entering the droplet over an annular ring are focused at the turning point rather than along a line. The method of Ref. 4 is employed in Appendix B to calculate the density of rays on the axial caustics. We find that the ray-theory intensity of the $p$-ray axial caustics is given by

$$
\frac{\mathrm{d} F_{p}}{\mathrm{~d} z}=\left|\frac{2 \pi a^{2} n \kappa T\left(\theta_{i}\right) R^{p-1}\left(\theta_{i}\right) \sin \left(\theta_{i}\right) \cos \left(\theta_{r}\right) \sin ^{2}(\gamma)}{\cos (\gamma) \sin \left(\theta_{r}\right)+\kappa \sin (\gamma) \cos \left(\theta_{r}\right)}\right|,
$$




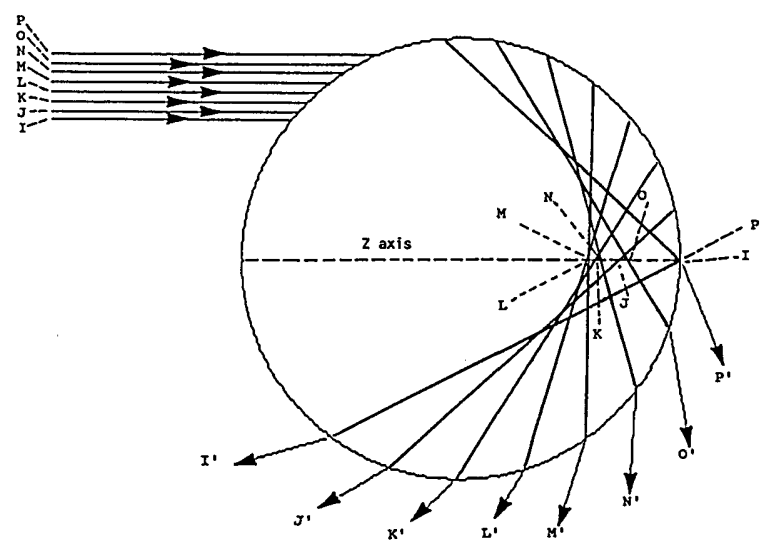

scattering to isolate one component of the far-field intensity from interference effects produced between it and all other components and to suggest physical mechanisms for various scattering phenomena. ${ }^{13-22}$ It also provides a theoretical justification for the physical-optics model for light scattering from bubbles. ${ }^{23-26}$

With the use of the notation of Ref. 13, the Debye series expansions of the Mie theory partial-wave amplitudes $a_{l}$ and $b_{l}$ for the scattered fields are

$$
\begin{aligned}
{\left[\begin{array}{l}
a_{l} \\
b_{l}
\end{array}\right] } & =\frac{1}{2}\left(1-R_{l}^{22}-\frac{T_{l}^{21} T_{l}^{12}}{1-R_{l}^{11}}\right) \\
& =\frac{1}{2}\left[1-R_{l}^{22}-\sum_{p=1}^{\infty} T_{l}^{21}\left(R_{l}^{11}\right)^{p-1} T_{l}^{12}\right],
\end{aligned}
$$

and the Debye-series expansions of the Mie-theory partialwave amplitudes $c_{l}$ and $d_{l}$ for the interior fields are

$$
\left[\begin{array}{l}
c_{l} \\
d_{l}
\end{array}\right]=\frac{T_{l}^{21}}{1-R_{l}^{11}}=\sum_{p=1}^{\infty} T_{l}^{21}\left(R_{l}^{11}\right)^{p-1} .
$$

In these expansions region 1 is the interior of the sphere, region 2 is outside the sphere, $T_{l}^{21}$ is the amplitude transmission coefficient for the $l$ spherical multipole partial wave to be transmitted from region 2 into region $1, R_{l}{ }^{11}$ is the amplitude reflection coefficient for the $l$ partial wave to be internally reflected from region 1 back into the same region, and

$$
\begin{aligned}
T_{l}^{21} & =\frac{-2 i / x^{2}}{\left(t_{l}{ }^{1}+t_{l}^{2}\right)+i\left(t_{l}^{3}-t_{l}^{4}\right)}, \\
R_{l}{ }^{11} & =\frac{-\left(t_{l}{ }^{1}-t_{l}^{2}\right)-i\left(t_{l}^{3}+t_{l}^{4}\right)}{\left(t_{l}{ }^{1}+t_{l}^{2}\right)+i\left(t_{l}^{3}-t_{l}{ }^{4}\right)} .
\end{aligned}
$$

For $a_{l}$ and $c_{l}$, the $t_{l}^{i}$ are given by

$$
\begin{aligned}
t_{l}= & x y\left\{j_{l}(x) j_{l-1}(y)-n j_{l-1}(x) j_{l}(y)\right. \\
& \left.+\left[l\left(n^{2}-1\right) / y\right] j_{l}(x) j_{l}(y)\right\}, \\
t_{l}{ }^{2}= & x y\left\{n_{l}(x) n_{l-1}(y)-n n_{l-1}(x) n_{l}(y)\right. \\
& \left.+\left[l\left(n^{2}-1\right) / y\right] n_{l}(x) n_{l}(y)\right\}, \\
t_{l}{ }^{3}= & x y\left\{n_{l}(x) j_{l-1}(y)-n n_{l-1}(x) j_{l}(y)\right. \\
& \left.+\left[l\left(n^{2}-1\right) / y\right] n_{l}(x) j_{l}(y)\right\}, \\
t_{l}{ }^{4}= & x y\left\{j_{l}(x) n_{l-1}(y)-n j_{l-1}(x) n_{l}(y)\right. \\
& \left.+\left[l\left(n^{2}-1\right) / y\right] j_{l}(x) n_{l}(y)\right\},
\end{aligned}
$$

and, for $b_{l}$ and $d_{l}$, the $t_{l}{ }^{i}$ are given by

$$
\begin{aligned}
t_{l}^{1} & =x y\left[n j_{l}(x) j_{l-1}(y)-j_{l-1}(x) j_{l}(y)\right], \\
t_{l}^{2} & =x y\left[n n_{l}(x) n_{l-1}(y)-n_{l-1}(x) n_{l}(y)\right], \\
t_{l}^{3} & =x y\left[n n_{l}(x) j_{l-1}(y)-n_{l-1}(x) j_{l}(y)\right], \\
t_{l}^{4} & =x y\left[n j_{l}(x) n_{l-1}(y)-j_{l-1}(x) n_{l}(y)\right],
\end{aligned}
$$

where

$$
\begin{aligned}
& x=2 \pi a / \lambda, \\
& y=n x
\end{aligned}
$$

and where $j_{l}$ and $n_{l}$ are spherical Bessel functions and spherical Neumann functions, respectively. (These are 
also known as spherical Bessel functions of the first and second kinds:) In addition, the amplitude transmission and reflection coefficients satisfy

$$
\begin{aligned}
\left|R_{l}{ }^{22}\right| & =\left|R_{l}{ }^{11}\right|, \\
T_{l}^{12} & =T_{l}^{21} / n, \\
\left|T_{l}^{21}\right|^{2} / n+\left|R_{l}{ }^{11}\right|^{2} & =1 .
\end{aligned}
$$

The physical interpretations of the various terms in Eq. (10) are given in Ref. 13. For the interior of the droplet, the $p$ term in Eq. (11) corresponds to spherical multipole partial waves that have entered the droplet and have made $p-1$ internal reflections. The Mie-theory equivalent of the $p$-ray family of Section 2 is the evaluation of the internal fields employing the $p$ term in Eq. (11) for $c_{l}$ and $d_{l}$.

Over the years, one concern in numerical Mie-theory computations has been the determination of the partial wave $l_{\max }$ at which the Mie series is truncated. For farfield scattering Dave's criterion ${ }^{27}$ is that the Mie scattering amplitudes $a_{l}$ and $b_{l}$ satisfy $\left|a_{l_{\max }}\right|^{2}+\left|b_{l_{\max }}\right|^{2} \leq 10^{-14}$. Occasional numerical difficulties with this criterion and the need for faster Mie calculation algorithms led Wiscombe to improve the criterion ${ }^{28}$ to $l_{\max }=x+4.05 x^{1 / 3}+$ 2 , which ensures that $a_{l_{\max }}$ and $b_{l_{\max }}$ are each of order $10^{-8}$. For internal-source-function calculations previous authors have used either the first ${ }^{29,30}$ or the second ${ }^{31,32}$ criterion. However, each of these criteria leads to less precision for interior-field calculations than for far-field-scattering calculations. This can be seen from the Debye series as follows. For high partial waves (i.e., $l>x$ ) we have $T_{l}^{21} \rightarrow 0$ and $R_{l}{ }^{11} \rightarrow 1$. Assume that, for such a partial wave,

$$
T_{l}^{21} \sim \epsilon \ll 1 \text {. }
$$

Then, substituting relation (21) into Eq. (20) and Taylor series expanding, we get

$$
R_{l}^{11} \sim 1-\left(\epsilon^{2} / 2 n\right) .
$$

From Eq. (11) the interior Mie amplitudes are of order

$$
c_{l} \sim d_{l} \sim \epsilon,
$$

while from Eq. (10) the scattering Mie amplitudes are of order

$$
a_{l} \sim b_{l} \sim \epsilon^{2} .
$$

As a result, the Mie interior amplitudes that are calculated with $c_{l}$ and $d_{l}$ converge more slowly than the Mie scattering amplitudes that are calculated with $a_{l}$ and $b_{l}$. To obtain the same precision for interior-field computations that one demands for far-field-scattering computations, one must employ more terms in the Mie series than indicated by Dave's or Wiscombe's criterion. A related situation occurs when one is searching for morphological scattering resonances in the far-field-scattering amplitudes. ${ }^{24,33}$ Using the formalism of Ref. 34, we find that the series cutoff

$$
l_{\max }=x+7 x^{1 / 3}+2
$$

ensures that $c_{l_{\max }}$ and $d_{l_{\max }}$ are of the order $10^{-8}$ for $35 \leq$ $x \leq 10,000$ and for $\operatorname{Im}(n) \leq 0.1$.

The cutoff of Eq. (25) was used with the internal-field formulas of Refs. 5 and 6 to determine the source function of a sphere

$$
S(\mathbf{r})=\left[\mathbf{E}^{*}(\mathbf{r}) \cdot \mathbf{E}(\mathbf{r})\right] / E_{0}^{2},
$$

where $E_{0}$ is the incident field strength, for size parameter

$$
x=100.0
$$

and real refractive index

$$
n=1.36
$$

illuminated by unpolarized plane waves. The spherical Bessel functions that occur in the radial dependence of the electric field and in the evaluation of $c_{l}$ and $d_{l}$ were computed by downward recursion. The spherical Neumann functions were computed by upward recursion.

The full Mie interior source function is shown in Fig. 5 in three different formats. Figure 5 (a) is a $100 \times 100$

(a)

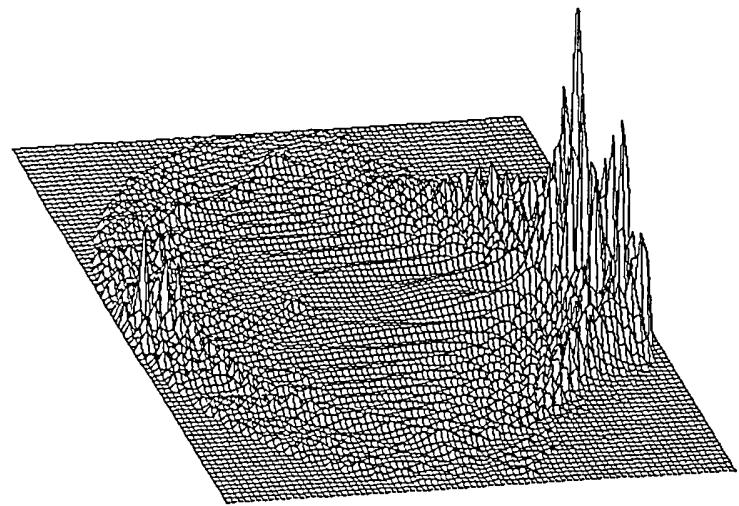

(b)

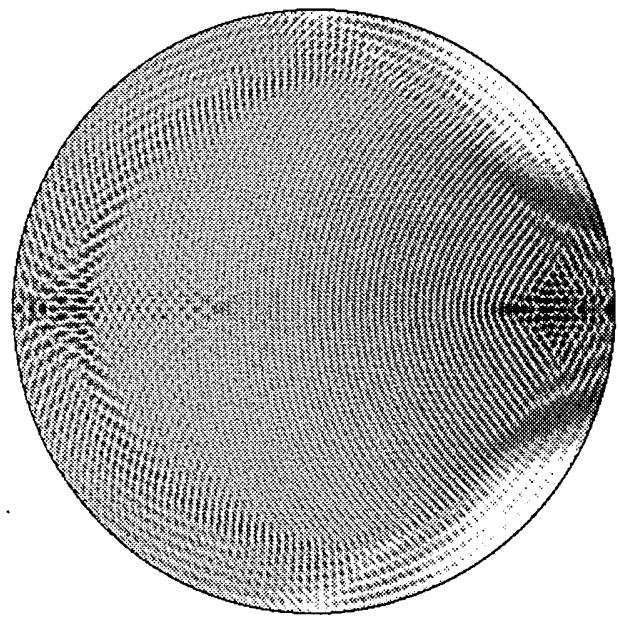

(c)

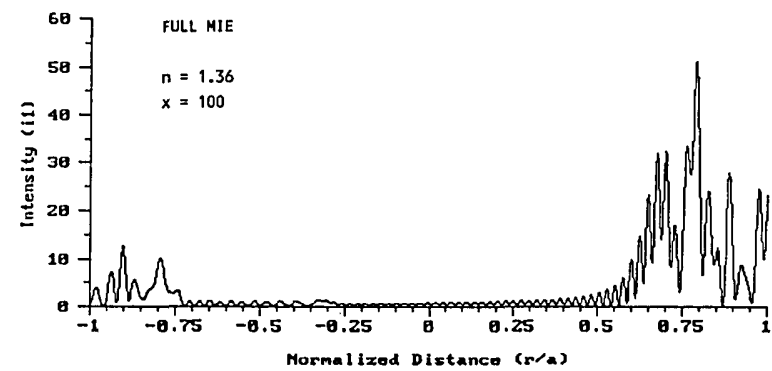

Fig. 5. Interior source function in Mie theory for a sphere with $x=100, n=1.36$, and for unpolarized incident light shown (a) as a carpet plot, (b) as a gray-level plot, and (c) along the $Z$ axis of the droplet. 


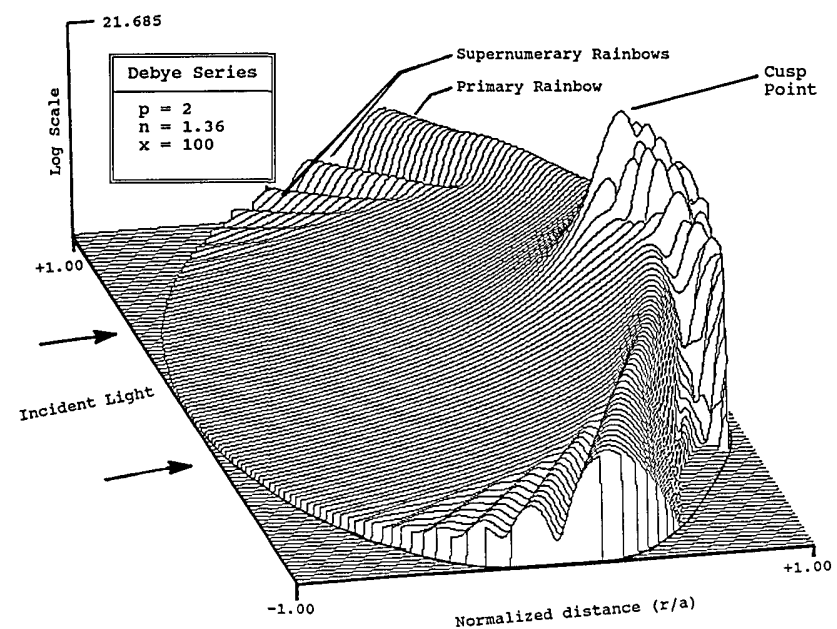

Fig. 6. The $p=2$ Debye-series contribution to the interior source function showing the $p=2$ cusp point and the interior portion of the first-order rainbow and supernumerary rainbows.

point carpet plot and shows the strong interior-field enhancements on the sphere's axis at $r \approx \pm 0.8 a$. These enhancements in the source function are compatible with the locations of the enhancements found in previous Mietheory calculations ${ }^{19,35-41}$ and will be addressed in Section 4. Figure 5(b) is the same source function calculated on a $500 \times 500$ point grid and logarithmically mapped into 64 shades of gray, black indicating the most intense fields and white the least intense. This gray-level plot emphasizes the interference structure of the interior source function. Figure 5(c) shows the source function on the sphere axis. The enhancements at $r \approx \pm 0.8 a$ appear to be a number of closely spaced individual enhancements.

The contribution to the source function provided by the individual $p=1$ to $p=8$ terms of the Debye series of Eq. (11) was also calculated for unpolarized incident light and is shown as the gray-level plots in Fig. 2 along with the ray-theory predictions. A common rule of thumb has been that ray theory becomes a good approximation to the far-field primary-rainbow caustic for $x \geq 2000 .^{42}$ Furthermore, when rainbows of higher order are observed, larger values of $x$ are required before these rainbows are well formed. ${ }^{27}$ For the interior caustic structure of Fig. 2, ray theory is already a qualitatively good approximation to wave theory at $x=100$ for all values of $p \leq 8$.

Figure 2 shows a number of other interesting features. As with all other wave-theory optical caustics, ${ }^{43}$ the infinite intensity of ray-theory caustics is rendered finite and is surrounded by diffraction and interference structure. For $p=2$ the interior portion of the first-order rainbow is visible in both the ray picture and the Debyeseries gray-level plot. It begins somewhat above and below the $p=2$ cusp point and extends to the sphere surface, where it continues into the far field to become the familiar first-order rainbow. This behavior is shown more visibly in the $p=2$ carpet plot of Fig. 6 . Also surprisingly visible in Fig. 6 are the first few supernumerary rainbows inside the droplet. One other feature of note in Fig. 2 is how the interference structure in the gray-level plots corresponds to regions of intersecting rays in the line points. For example, the gray-level plot for $p=3$ shows much interference structure on the illuminated side of droplet (from $r \approx-1.0 a$ to $r \approx-0.27 a$ ) and a smooth decrease in intensity on the shadow side (from $r \approx-0.27 a$ to $r \approx$
$+1.0 a$ ). The corresponding line plot for $p=3$ shows many rays intersecting one another in the region on the illuminated side and the rays diverging in the region on the shadow side.

In order to assess the quantitative agreement between the caustics of ray theory and the individual terms of the Debye-series expansion of the interior electric field, we calculated the source function along the $Z$ axis for the $p=$ 1 to $p=10$ terms of the Debye series. The results are shown in Fig. 7 as the solid curves. It is remarkable that, for large $p$, the source function for an individual term in the Debye series ranges over as many as 16 orders of magnitude. This large variation is easily understood. From Section 2 the intensity of a $p$ ray is proportional to $T\left(\theta_{i}\right) R^{p-1}\left(\theta_{i}\right)$. Consider the refractive index of Eq. (28) and $p=10$. The intensity at the center of the sphere is due to rays incident near the sphere axis with $\theta_{i} \approx 0, T \approx$ $0.977, R \approx 0.023$, and $T R^{9} \approx 10^{-15}$. But the intensity near the surface of the sphere is due to rays near grazing incidence with $\theta_{i} \approx \pi / 2$ and perhaps with $T \approx 0.01$ and $R \approx 0.99$ giving $T R^{9} \approx 10^{-2}, 13$ orders of magnitude larger than at the center. In other words, rays near grazing incidence have a low probability of being transmitted into the droplet. But, once they are inside, the probability of their surviving many internal reflections is quite large. On the other hand, rays near normal incidence are likely to be transmitted into the droplet. But, once inside, they are again likely to be transmitted back out. Since the cusp points of the $p$-ray caustics are due to rays near normal incidence, this argument explains why they are the locations on the caustics of nearly the weakest intensity, rather than the strongest. The illusion in the ray-theory pictures of Fig. 2 that the cusp point is the most intense location on the caustic results from incorrectly drawing all the ray lines with equal darkness or thickness. Since $T\left(\theta_{i}\right) R^{p-1}\left(\theta_{i}\right)$ continues to grow in going from normal incidence to grazing incidence, the axial caustics become more intense as they progress from the center of the droplet to the perimeter.

In Section 2 we argued that, on the $Z$ axis, the electric field should be dominated by the contribution provided by the axial caustics. Figure 7 shows the density of rays on the axial caustics (data displayed as points) given by Eq. (9) multiplied by a single scaling factor for all $p$. The locations on the curves that are multivalued represented regions in which the axial caustic has turned back on itself. As this figure shows, at $x=100$ the ray-theory model of caustics within a liquid droplet is a quantitatively good approximation to the caustic structure predicted by wave theory. At the turning points of the axial caustics, the ray-theory intensity becomes infinite, while the Debyeseries intensity undergoes a rapid increase by a number of orders of magnitude and is accompanied on one side by an interference structure, a behavior typical of diffraction caustics. ${ }^{43}$ For larger $x$ the interference structure becomes finer and the caustics become quite well defined.

\section{COMPARISON WITH STIMULATED RAMAN SCATTERING AND FLUORESCENCE EMISSION}

The transition rates for SRS and fluorescence emission at a given location within a sample are proportional to the square of the stimulating electric field at that location, 

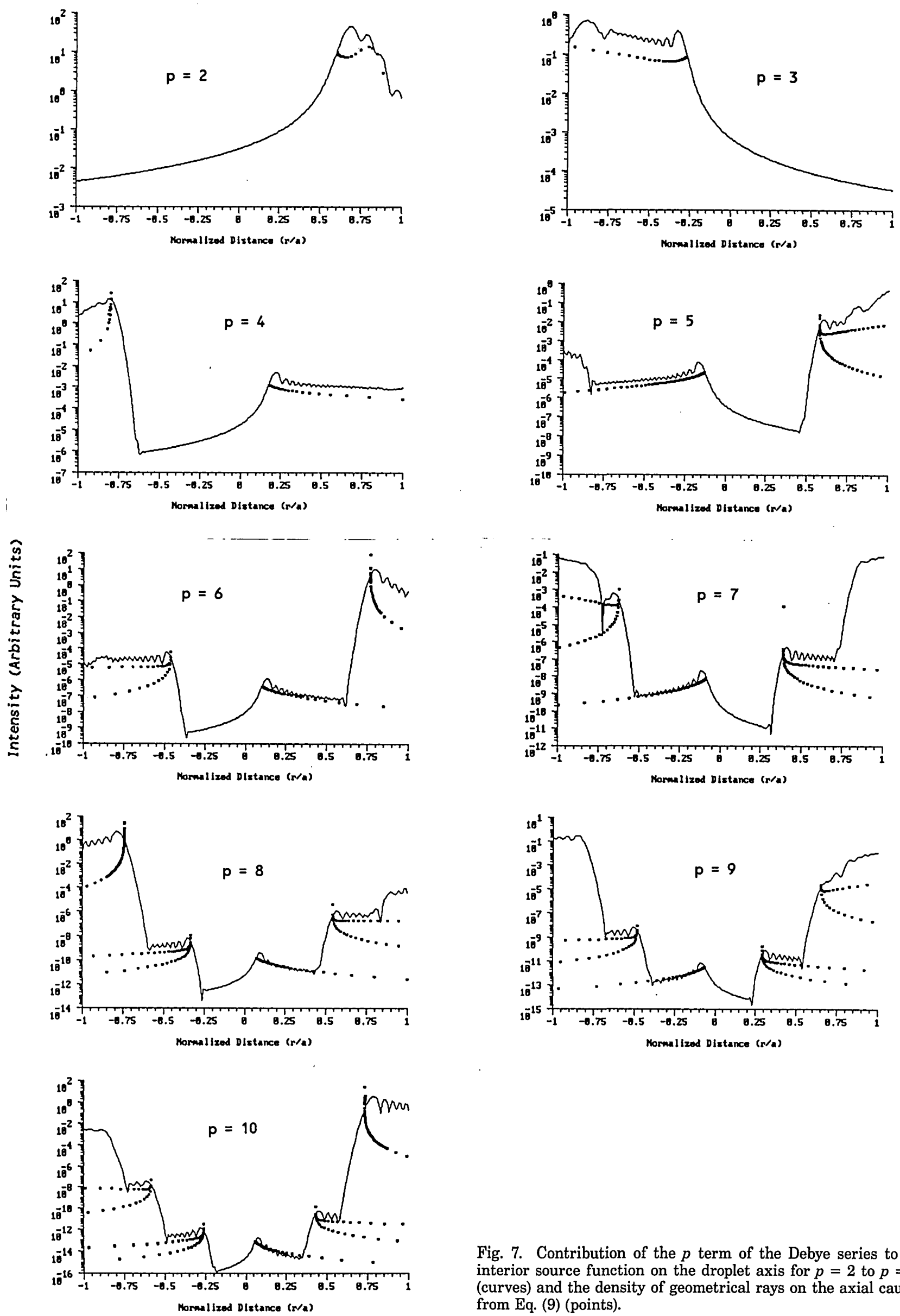

Fig. 7. Contribution of the $p$ term of the Debye series to the interior source function on the droplet axis for $p=2$ to $p=10$ (curves) and the density of geometrical rays on the axial caustic from Eq. (9) (points). 


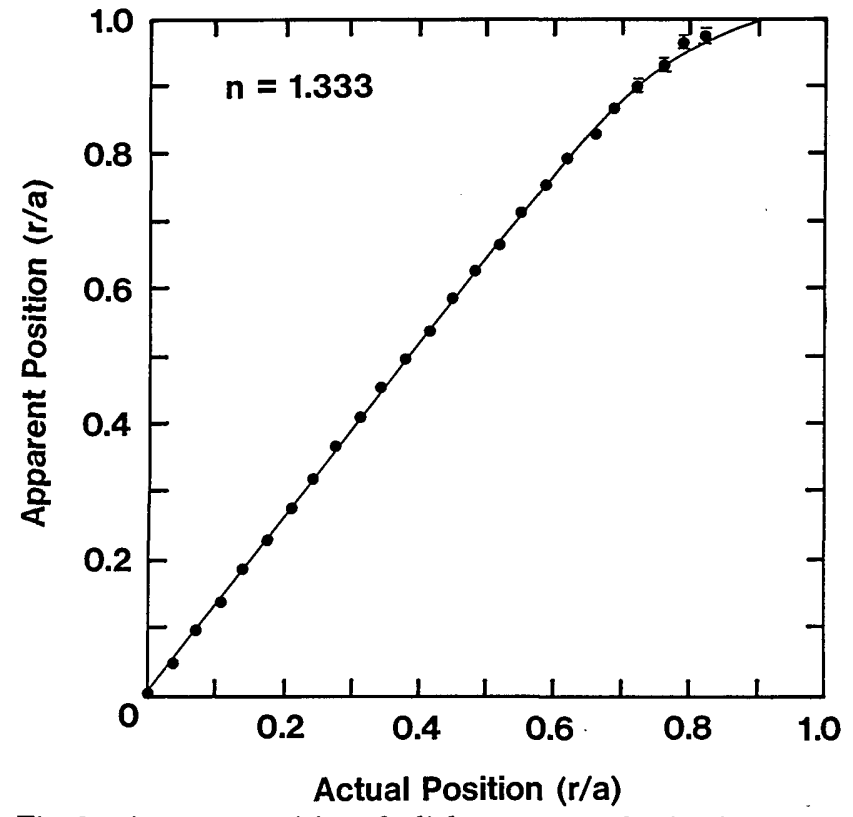

Fig. 8. Apparent position of a light source on the droplet axis as a function of the actual position for the geometric-optics model of Appendix $\mathrm{C}$ and $n=1.333$. The filled-circle data points are the measured positions of grid marks on a scale fitted along the diameter of an 18.4-cm thin-walled glass globe when empty (actual positions) and filled with water (apparent positions).

i.e., the source function. ${ }^{44}$ Exploiting this fact, these inelastic light-scattering processes have been used to probe the distribution of electric-field strength within spherical liquid droplets illuminated by laser light..$^{35,36,45,46}$ Three regions of enhanced source function within the droplet have been observed. The first region is on the droplet's shadow side at $r \approx+0.8 a$. The second region is on the droplet's illuminated side at $r \approx-0.8 a$. The third region is the entire circumference of the droplet. This last region of strong emission, in contrast to the first two, is observed only for droplets of certain diameters and corresponds to the situation for which the diameter of the droplet, its refractive index, and the wavelength of the incident light satisfy the conditions for the occurrence of a morphological scattering resonance. ${ }^{47-49}$

The analysis of Sections 2 and 3 and Fig. 7 can be used to explain the first region of enhanced source function on the droplet's shadow side. The regions of most intense focusing on the shadow side of the droplet axis in Fig. 7 occur at focal point of the $p=2$ rays and also at the maxima of the Debye-series interference patterns associated with the outermost turning points of the axial caustics at $r \approx+0.80 a$ for $p=6$ and $r \approx+0.78 a$ for $p=10$.
Other turning points in this region are the outermost turning points of the $p=5$ and $p=9$ axial caustics. But these are lower than those for the $p=6$ and $p=10$ by approximatly 2-5 orders of magnitude. As a result we attribute the $r \approx+0.8 a$ source of SRS and fluorescence emission to the $p=2$ focal point amplified by the near-identical turning points of the $p=6,10,14, \ldots$, axial caustics.

In Refs. 35, 36, and 45 the shadow-side source of SRS and fluorescence emission seems to be on the droplet axis. However, in Ref. 46 it appears instead on the surface of the droplet at the position of the $p=1$ caustic ring. It is pointed out in Refs. 44, 50, and 51 that the electric- and magnetic-field boundary conditions at the surface of the droplet must be satisfied not only by the radiation at the incident wavelength but also by the radiation at the inelastically scattered wavelength. This condition causes the inelastically scattered radiation to be preferentially emitted in certain directions and causes these directions to vary for stimulated molecules at different locations within the droplet. Since the observations of Refs. 35, 36, and 45 and those of Ref. 46 were made at different scattering angles, this directionality of the inelastically scattered radiation may provide the explanation for the difference between the observations. This point, however, requires further study.

The second region of SRS and fluorescence emission on the illuminated side of the droplet (at $r \approx-0.8 a$ ) is said to be caused by the focusing on the axis of rays that have internally reflected twice within the droplet ${ }^{37}$ (i.e., $p=$ 3 rays). This interpretation is shown in Figs. 2 and 7 to be incorrect since, for $n=1.36$, Eqs. (5) support the prediction that the $p=3$ cusp point should occur at $z=$ $-0.27 a$. This result corresponds to the small enhancement on the full Mie plot in Fig. 5 (c) at $z \approx-0.27 a$. The locations of the outermost turning points of the axial caustics at $r \approx-0.80 a$ for $p=4$ and at $r \approx-0.78 a$ for $p=8$ closely correspond to the maximum source function enhancement in Fig. 5(c). As a result we attribute the $r \approx$ $-0.8 a$ source to the nearly identical locations of the turning points of the $p=4,8,12, \ldots$, axial caustics.

It has been noted that, when one is observing the sources of SRS and fluorescence emission within liquid droplets, the curvature of the droplet surface causes the apparent source of the emission to be shifted from its actual location. ${ }^{35,52}$ This shift in position is calculated in Appendix $\mathrm{C}$ from geometrical optics and is shown in Fig. 8 for $n=1.333$. Also shown in Fig. 8 are the results of an experimental verification of the position shift. The 18.4cm-diameter thin-wall glass globe described in Section 2 was fitted with a scale along its diameter. The globe was photographed from the side, then filled with water and

Table 1. Experimentally Observed Locations of the Sources of Stimulated Raman Scattering and Fluorescence Emission in Liquid Droplets

\begin{tabular}{|c|c|c|c|c|}
\hline Reference & Liquid & Droplet Radius & Shadow-Side Enhancement & Illuminated-Side Enhancement \\
\hline 35 & $\begin{array}{l}\text { Ethanol } \\
\qquad n=1.36\end{array}$ & $35 \mu \mathrm{m}$ & $0.66 a \leq r \leq 0.79 a$ & $-0.78 a \leq r \leq-0.70 a$ \\
\hline 36 & $\begin{array}{l}\text { Methanol } \\
\quad n=1.33\end{array}$ & $25 \mu \mathrm{m}$ & $0.67 a \leq r \leq 1.00 a$ & $-1.00 a \leq r \leq-0.63 a$ \\
\hline 45 & $\begin{array}{l}\text { Ethanol }^{a} \\
\quad n=1.36\end{array}$ & Not given & $0.42 a \leq r \leq 1.00 a$ & $-1.00 a \leq r \leq-0.26 a$ \\
\hline
\end{tabular}


photographed again. The shifts in the positions of the grid marks on the scale between the two photographs were measured. The agreement with the results of Appendix $\mathrm{C}$ is good.

We applied the method of Appendix $\mathrm{C}$ to determine the actual locations of the sources of SRS and fluorescence emission in the photographic observations of Refs. 35, 36, and 45. The results are given in Table 1. The experimental results agree with the locations of the $p=2$ cusp point and the outermost turning points of the $p=4$, $6,8,10, \ldots$, axial caustics found in Fig. 7 .

\section{CONCLUSIONS}

In the past the Debye-series expansion of the far-field Mie scattering amplitudes has provided a valuable method by which a scattering mechanism can be computationally isolated from all the other effects that interfere with it. In this paper we show that the Debye-series expansion of the interior-field Mie amplitudes has a physical interpretation of equal richness. The individual terms of the Debye series correspond to families of rays inside the droplet. The caustics of ray theory are reproduced by the Debyeseries calculations and decorated with diffraction structure typical of wave-theory treatments. Further, the Debye-series analysis provides the physical explanation of the source-function hot spots observed with inelastic light scattering.

\section{APPENDIX A}

Consider the $p$ ray that enters the liquid droplet with the angle of incidence $\theta_{i}$ at the coordinates

$$
\begin{aligned}
& z_{i}=-a \cos \left(\theta_{i}\right), \\
& \rho_{i}=+a \sin \left(\theta_{i}\right) .
\end{aligned}
$$

The location where this ray intersects the sphere surface at the $p-1$ internal reflection is

$$
\begin{aligned}
& z_{p-1}=-a \cos \left[\theta_{i}+2(p-1) \phi\right], \\
& \rho_{p-1}=+a \sin \left[\theta_{i}+2(p-1) \phi\right],
\end{aligned}
$$

and the location where it intersects the sphere surface at the $p$ internal reflection is

$$
\begin{gathered}
z_{p}=-a \cos \left(\theta_{i}+2 p \phi\right), \\
\rho_{p}=+a \sin \left(\theta_{i}+2 p \phi\right),
\end{gathered}
$$

where

$$
\phi=(\pi / 2)-\theta_{r}
$$

and $\theta_{r}$ is the angle of refraction given in Eq. (2). The equation of this $p$ ray is

$$
\rho=\rho_{p-1}-[\tan (\gamma)]\left(z-z_{p-1}\right),
$$

where $\gamma$ is given in Eq. (3). Consider the intersection of this ray with the $p$ ray whose angle of incidence is $\theta_{i}+\epsilon$. The angle of refraction of this second $p$ ray is $\theta_{r}+\delta$, where

$$
\delta \approx \frac{\epsilon \cos \left(\theta_{i}\right)}{n \cos \left(\theta_{r}\right)}
$$

for small $\epsilon$. In the limit as $\epsilon \rightarrow 0$, the intersection point of the first and second $p$ rays is the location on the caustic given by Eqs. (1).

For the axial caustic, consider the location where the $p$ ray crosses the $Z$ axis. Equation (A5) with $\rho=0$ gives

$$
z=z_{p-1}+\frac{\rho_{p-1}}{\tan (\gamma)}
$$

Substituting Eqs. (A2) into Eq. (A7) gives Eqs. (8).

\section{APPENDIX B}

Let $\mathrm{d} F_{\text {inc }}$ be the flux incident upon the annular ring of width $\mathrm{d} \theta_{i}$ centered at the angle of incidence $\theta_{i}$, and let $\mathrm{d} z$ be the progress along the $p$-ray axial caustic that results from increasing the angle of incidence from $\theta_{i}-\mathrm{d} \theta_{i} / 2$ to $\theta_{i}+\mathrm{d} \theta_{i} / 2$. Then

$$
\mathrm{d} F_{\text {inc }}=2 \pi a^{2} \sin \left(\theta_{i}\right) \cos \left(\theta_{i}\right) \mathrm{d} \theta_{i} .
$$

The fraction of this incident flux $\mathrm{d} F$ that survives $p-1$ internal reflections is

$$
\mathrm{d} F_{p}=\mathrm{d} F_{\mathrm{inc}} T\left(\theta_{i}\right) R^{p-1}\left(\theta_{i}\right) .
$$

Then, following the procedure of Ref. 4,

$$
\frac{\mathrm{d} F_{p}}{\mathrm{~d} z}=\left(\frac{\mathrm{d} F_{p}}{\mathrm{~d} \theta_{i}}\right) /\left(\frac{\mathrm{d} z}{\mathrm{~d} \theta_{i}}\right) .
$$

The expression for $z$ is given in Eqs. (8). Differentiating it with respect to $\theta_{i}$ and inserting the result into Eq. (B3) give Eq. (9).

\section{APPENDIX C}

Consider a point on the axis of the sphere at the location

$$
z_{s}=w a
$$

as in Fig. 9(a). Assume that this point source emits light rays in all directions. Consider the light ray that leaves the source at an angle $\alpha$ with respect to the $\rho$ axis. Then the $z$ coordinate of the intersection of this light ray with the surface of the sphere is

$$
z_{\text {out }}=u a \text {, }
$$

where

$$
\begin{aligned}
u & =w+\left[w^{2}+1-2 w \cos (\beta)\right]^{1 / 2} \cos \left(\beta+\theta_{i}\right), \\
\sin \left(\theta_{i}\right) & =\frac{w \sin (\beta)}{\left[w^{2}+1-2 w \cos (\beta)\right]^{1 / 2}} .
\end{aligned}
$$

The angle that the exiting ray makes with the $Z$ axis is

$$
\xi=\beta+\theta_{r}
$$

where

$$
\sin \left(\theta_{r}\right)=\frac{n w \sin (\beta)}{\left[w^{2}+1-2 w \cos (\beta)\right]^{1 / 2}} .
$$

As is shown in Fig, 9(b), many such light rays leave the source $S$ and pass through a lens. These rays are then imaged at the point $\overline{\mathrm{S}}$ on a viewing screen parallel to the $Z$ axis, as shown in Fig. 9(c). These rays, such as rays A, 


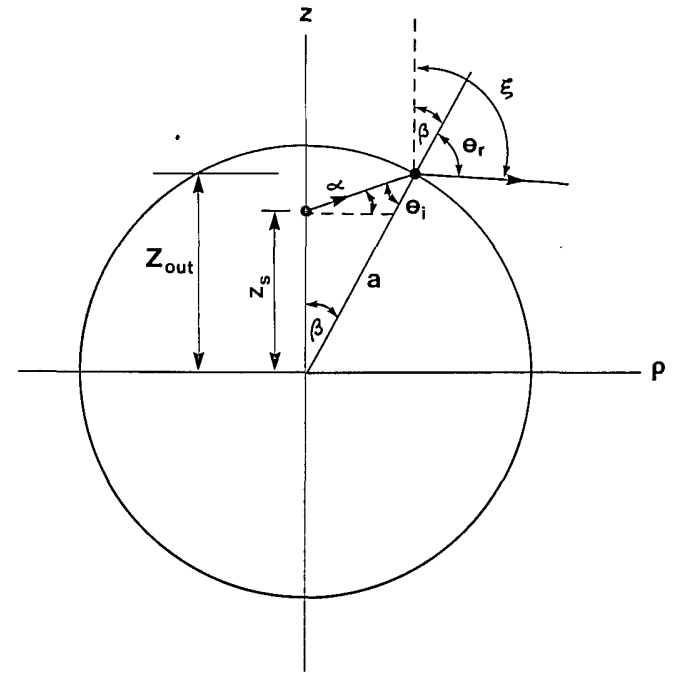

(a)

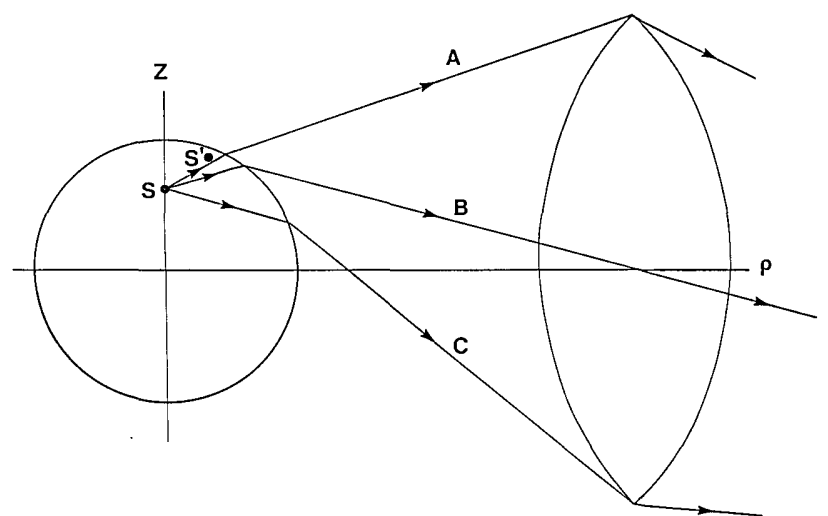

(b)

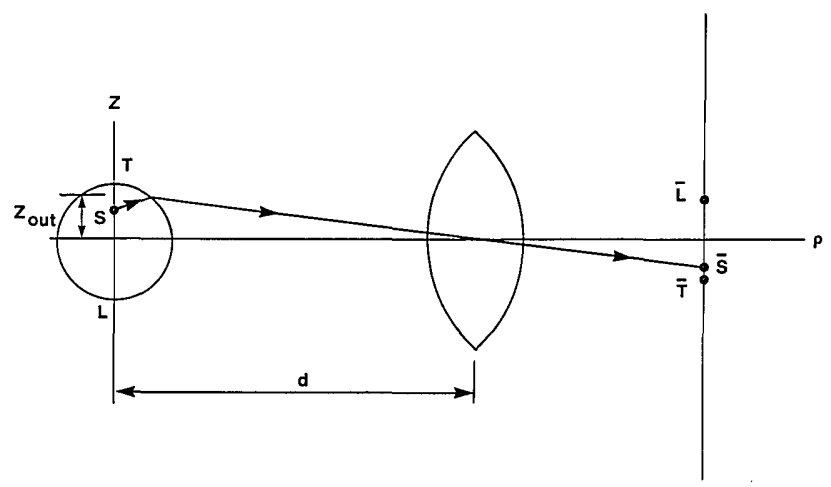

(c)

Fig. 9. (a) Light ray leaving a source at the coordinate $z_{s}$ on the axis of a spherical droplet. (b) Three such rays leaving source $S$ and imaged by a lens. The virtual source of rays $A, B$, and $C$ is $\mathrm{S}^{\prime}$. (c) Imaging of the spherical droplet by a lens. The image of the topmost portion of droplet $\mathrm{T}$ is $\overline{\mathrm{T}}$. The image of the lowermost portion of droplet $L$ is $\bar{L}$. The image of source $S$ is $\bar{S}$.

B, and C in Fig. 9(b), appear to originate from the virtual source $\mathrm{S}^{\prime}$ within the droplet. Consider ray $\mathrm{B}$, which passes through the center of the lens. If $d$ is the distance from the droplet to the lens, then from Fig. 9(c) the angle that this ray makes with the $Z$ axis is approximately

$$
\xi=(\pi / 2)+\arctan \left(z_{\text {out }} / d\right) .
$$

In the limit $d \gg a \geq z_{\text {out }}$, combining Eqs. (C5) and (C7) gives

$$
\cos (\beta)=\sin \left(\theta_{r}\right)
$$

or

$$
w^{2}+1-2 w \cos (\beta)=n^{2} w^{2} \tan ^{2}(\beta) .
$$

Finally, given the actual position of the source $w$, one numerically solves Eq. (C9) to obtain $\beta$. One then inserts $\beta$ into Eq. (C4) to find $\theta_{i}$ and substitutes the result into Eq. (C3) to find $u$. If $a \ll d$, this result is also the apparent position of the virtual source $\mathrm{S}^{\prime}$.

\section{ACKNOWLEDGMENT}

This research was carried out while J. A. Lock was a National Aeronautics and Space Administration/ASEE Summer Faculty Fellow in the Optical Measurement Systems Branch of the NASA Lewis Research Center.

Note added in proof: The results of this paper may also serve as a standard to which the method of Ref. 54 may be compared when that work is extended to two dielectric interfaces.

\section{REFERENCES}

1. J. R. Reitz, F. J. Milford, and R. W. Christy, Foundations of Electromagnetic Theory, 3rd ed. (Addison-Wesley, Reading, Mass., 1979), Subsec. 18-2.

2. M. Herzberger, Modern Geometrical Optics (Wiley Interscience, New York, 1958), p. 156.

3. T. Pearcy, "The structure of an electromagnetic field in the neighborhood of a cusp caustic," Philos. Mag. 37, 311-317 (1946).

4. D. G. Burkhard and D. L. Shealy, "Formula for the density of tangent rays over a caustic surface," Appl. Opt. 21, 32993306 (1982).

5. P. W. Dusel, M. Kerker, and D. D. Cooke, "Distribution of absorption centers within irradiated spheres," J. Opt. Soc. Am. 69, 55-59 (1979).

6. W. M. Greene, R. E. Spjut, E. Bar-Ziv, A. F. Sarofim, and J. P. Longwell, "Photophoresis of irradiated spheres: absorption centers," J. Opt. Soc. Am. B 2, 998-1004 (1985).

7. C. C. Dobson and J. W. L. Lewis, "Survey of the Mie problem source function," J. Opt. Soc. Am. A 6, 463-466 (1989).

8. W. M. Greene, R. E. Spjut, E. Bar-Ziv, A. F. Sarofim, and J. P. Longwell, "Photophoresis of irradiated spheres: absorption centers: errata," J. Opt. Soc. Am. B 5, 866 (1988).

9. H. C. van de Hulst, Light Scattering by Small Particles (Dover, New York, 1981), Subsec. 9.22.

10. Ref. 1, Subsec, 18-5.

11. H. M. Nussenzveig, "High-frequency scattering by a transparent sphere. I. Direct reflection and transmission," J. Math. Phys. 10, 82-124 (1969).

12. H. M. Nussenzveig, "High-frequency scattering by a transparent sphere. II. Theory of the rainbow and the glory," J. Math. Phys. 10, 125-176 (1969).

13. J. A. Lock, "Cooperative effects among partial waves in Mie scattering," J. Opt. Soc. Am. A 5, 2032-2044 (1988).

14. V. Khare and H. M. Nussenzveig, "Theory of the rainbow," Phys. Rev. Lett. 33, 976-980 (1974).

15. V. Khare and H. M. Nussenzveig, "Theory of the glory," Phys. Rev. Lett. 38, 1279-1282 (1977).

16. V. Khare and H. M. Nussenzveig, "The theory of the glory," in Statistical Mechanics and Statistical Methods in Theory and Application, U. Landman, ed. (Plenum, New York, 1977), pp. 723-764.

17. H. M. Nussenzveig, "Complex angular momentum theory of the rainbow and the glory," J. Opt. Soc. Am. 69, 1068-1079; 1193-1194 (1979). 
18. H. M. Nussenzveig and W. J. Wiscombe, "Forward optical glory," Opt. Lett. 5, 455-457 (1980).

19. H. M. Nussenzveig and W. J. Wiscombe, "Efficiency factors in Mie scattering," Phys. Rev. Lett. 45, 1490-1494 (1980).

20. H. M. Nussenzveig and W. J. Wiscombe, "Diffraction as tunneling," Phys. Rev. Lett. 59, 1667-1670 (1987).

21. J. A. Lock, "Theory of the observations of high-order rainbows from a single water droplet," Appl. Opt. 26, 5291-5298 (1987).

22. J. A. Lock and J. R. Woodruff, "Non-Debye enhancements in the Mie scattering of light from a single water droplet," Appl. Opt. 28, 523-529 (1989).

23. P. L. Marston, "Critical angle scattering by a bubble: physical-optics approximation and observations," J. Opt. Soc Am. 69, 1205-1211 (1979).

24. P. L. Martson and D. L. Kingsbury, "Scattering by a bubble in water near the critical angle: inteference effects," J. Opt. Soc. Am. 71, 192-196 (1981).

25. D. S. Langley and P. L. Marston, "Critical angle scattering of laser light from bubbles in water: measurements, models, and application to sizing of bubbles," Appl. Opt. 23, 10441054 (1984).

26. W. P. Arnott and P. L. Marston, "Optical glory of small freely rising gas bubbles in water: observed and computed crosspolarized backscattering patterns," J. Opt. Soc. Am. A 5, 496-506 (1988).

27. J. V. Dave, "Subroutines for computing the parameters of the electromagnetic radiation scattered by a sphere," Rep. 3203237 (IBM Scientific Center, Palo Alto, Calif., 1968).

28. W. J. Wiscombe, "Improved Mie scattering algorithms," Appl. Opt. 19, 1505-1509 (1980).

29. G. J. Rosasco and H. S. Bennett, "Internal field resonance structure: implications for optical absorption and scattering by microscopic particles," J. Opt. Soc. Am. 68, 1242-1250 (1978).

30. A. Bott and W. Zdunkowski, "Electromagnetic energy within dielectric spheres," J. Opt. Soc. Am. A 4, 1361-1365 (1987).

31. C. C. Dobson and J. W. L. Lewis, "Survey of the Mie problem source function," J. Opt. Soc. Am. A 6, 463-466 (1989).

32. S. Chang, "Internal electromagnetic energy within a dielectric sphere in a plane-polarized TEM 00 laser beam," J. Opt. Soc. Am. B 6, 1332-1338 (1989).

33. S. C. Hill, C. K. Rushforth, R. E. Benner, and P. R. Conwell "Sizing dielectric spheres and cylinders by aligning measured and computed resonance locations: algorithm for multiple orders," Appl. Opt. 24, 2380-2390 (1985).

34. V. E. Cachorro and L. L. Salcedo, "New improvements for Mie scattering calculations," J. Electron. Waves Appl. (to be published)

35. D. S. Benincasa, P. W. Barber, J.-Z. Zhang, W.-F. Hsieh, and R. K. Chang, "Spatial distribution of the internal and nearfield intensities of large cylindrical and spherical scatterers," Appl. Opt. 26, 1348-1356 (1987).

36. R. G. Pinnick, P. Chýlek, M. Jarzembski, E. Creegan, V. Srivastava, G. Fernandez, J. D. Pendleton, and A. Biswas, "Aerosol-induced laser breakdown thresholds: wavelength dependence," Appl. Opt. 27, 987-996 (1988).
37. C. F. Wood, D. H. Leach, J.-Z. Zhang, R. K. Chang, and P. W. Barber, "Time-resolved shadowgraphs of large individual water and ethanol droplets vaporized by a pulsed $\mathrm{CO}_{2}$ laser," Appl. Opt. 27, 2279-2286 (1988).

38. A. B. Pluchino, "Surface waves and the raddiative properties of micron-sized particles," Appl. Opt. 20, 2986-2993 (1981).

39. P. Chýlek, J. D. Pendleton, and R. G. Pinnick, "Internal and near-surface scattered field of a spherical particle at resonant conditions," Appl. Opt. 24, 3940-3942 (1985).

40. P. Chýlek, M. A. Jarzembski, V. Srivastava, R. G. Pinnick, J. D. Pendleton, and J. P. Cruncleton, "Effect of spherical particles on laser-induced breakdown in gases," Appl. Opt. 26, 760-762 (1987)

41. J. P. Barton, D. R. Alexander, and S. A. Schaub, "Internal fields of a spherical particle illuminated by a tightly focused laser beam: focal point positioning effects at resonance," J. Appl. Phys. 65, 2900-2906 (1989).

42. Ref. 5, Subsec. 13-24.

43. M. V. Berry and C. Upstill, "Catastrophe optics: morphologies of caustics and their diffraction patterns," Prog. Opt. 18, 257-346 (1980).

44. M. Kerker and S. D. Druger, "Raman and fluorescent scattering by molecules embedded in spheres with radii up to several multiples of the wavelength," Appl. Opt. 18, 1172-1179 (1979)

45. P. Chýlek, M. A. Jarzembski, N. Y. Chou, and R. G. Pinnick, "The effect of size and material of liquid spherical particles on laser-induced breakdown," Appl. Phys. Lett. 49, 14751477 (1986).

46. M. A. Jarzembski and V. Srivastava, "Electromagnetic field enhancement in small liquid droplets using geometric optics," Appl. Opt. 28, 4962-4965 (1989).

47. P. Chýlek, "Partial wave resonances and the ripple structure in the Mie normalized extinction cross section," J. Opt. Soc. Am. 66, 285-287 (1976).

48. A. Ashkin and J. M. Dziedzic, "Observation of resonances in the radiation pressure on dielectric spheres," Phys. Rev. Lett. 38, 1351-1354 (1977).

49. A. Ashkin and J. M. Dziedzic, "Observation of optical resonances of dielectric spheres by light scattering," Appl. Opt. 20, 1803-1814 (1981)

50. H. Chew, P. J. McNulty, and M. Kerker, "Model for Raman and fluorescent scattering by molecules embedded in small particles," Phys. Rev. A 13, 396-404 (1976).

51. M. Kerker, P. J. McNulty, M. Sculley, H. Chew, and D. D. Cooke, "Raman and fluorescent scattering by molecules embedded in small particles: numerical results for incoherent optical processes," J. Opt. Soc. Am. 68, 1676-1686 (1978).

52. S. C. Hill and R. E. Benner, "Morphology-dependent resonances associated with stimulated processes in microspheres," J. Opt. Soc. Am. B 3, 1509-1514 (1986).

53. P. Chýlek, Department of Physics, Dalhousie University, Halifax B3H 3J5, Canada (personal communication)

54. Y. Ji and $\mathrm{K}$. Hongo, "Analysis of electromagnetic waves refracted by a spherical dielectric interface," J. Opt. Soc. Am. A 8, 541-548 (1991). 